Losada-Puente, L; Honrubia-Montesinos, C y Gil-Madrona, P. (2020) Validation of an Instrument of Professional Development in Infant Physical Education. Revista Internacional de Medicina y Ciencias de la Actividad Física y el Deporte vol. 20 (77) pp. 57-72 Http://cdeporte.rediris.es/revista/revista77/artvalidacion1108.htm

DOI: $10.15366 /$ rimcafd2020.77.004

\title{
ORIGINAL
}

\section{VALIDACIÓN DE UN INSTRUMENTO DE DESARROLLO PROFESIONAL EN EDUCACIÓN FÍSICA INFANTIL}

\section{VALIDATION OF AN INSTRUMENT OF PROFESSIONAL DEVELOPMENT IN INFANT PHYSICAL EDUCATION}

\author{
Losada-Puente, L. ; Honrubia-Montesinos, C. ${ }^{2}$ y Gil-Madrona, P. ${ }^{3}$ \\ 1 Doctora en Equidad e Innovación en Educación, Departamento de Didácticas Específicas y \\ Métodos de Investigación y Diagnóstico en Educación, Facultad de Educación, Universidade da \\ Coruña (España) luisa.losada@udc.es \\ 2 Doctorando en Humanidades, Artes y Educación, Departamento de Didáctica de Expresión \\ Artística, Musical y Corporal, Facultad de Educación de Albacete, Universidad de Castilla La \\ Mancha (España) Cristina.Honrubia@uclm.es \\ ${ }^{3}$ Doctor en Educación, Departamento de Didáctica de Expresión Plástica, Musical y Corporal, \\ Facultad de Educación de Albacete, Universidad de Castilla La Mancha (España) \\ pedro.gil@uclm.es
}

Código UNESCO/ UNESCO code: 5803 Preparación y Empleo de Profesores / Formation and employment of teachers

Clasificación Consejo de Europa: 5 Didáctica y metodología / Didactics and Methodology

Recibido 8 de marzo de 2018 Received March 8, 2018

Aceptado 3 de julio de 2018 Accepted July 3, 2018

\section{RESUMEN}

El objetivo consiste en construir y diseñar un cuestionario que evalúa el desarrollo profesional de los docentes de Educación Infantil en su vinculación con la Educación Física. Se han estudiado sus propiedades psicométricas con una muestra de 526 docentes mediante el programa estadístico SPSS v.23 y AMOS. Se llevaron a cabo análisis de confiabilidad, análisis factorial exploratorio, correlación ítem con puntaje total y análisis factorial confirmatorio. La consistencia del instrumento ha sido elevada $(\alpha=0,836)$. Además, hay una adecuada correlación ítem con puntaje total, y la presencia de siete factores en el análisis factorial exploratorio. El análisis factorial confirmatorio mostró un adecuado ajuste a los datos del modelo del cuestionario ( $\mathrm{X} 2 \mathrm{gl}=1,635$; $\mathrm{GFI}=0,929 ; \mathrm{CFI}=0,963 ; \mathrm{RMSEA}=0,039$ ). Los resultados sugieren que este cuestionario es un instrumento fiable y válido y se recomienda su uso en futuras investigaciones. 
PALABRAS CLAVE: Educación Física Infantil, Educación Infantil, docentes, desarrollo profesional, validación.

\begin{abstract}
The objective is to build and design a questionnaire that assesses the professional development of Early Childhood Education teachers in their connection with Physical Education. Their psychometric properties have been studied with a sample of 526 teachers through the statistical program SPSS v.23 and AMOS. Reliability analysis, exploratory factor analysis, item correlation with total score and confirmatory factor analysis were carried out. The consistency of the instrument has been high $(\alpha=0.836)$. In addition, there is an adequate item correlation with total score, and the presence of seven factors in the exploratory factor analysis. The confirmatory factor analysis showed an adequate adjustment to the model data of the questionnaire ( $\mathrm{X} 2 / \mathrm{gl}=1.635$, $\mathrm{GFI}=0.929, \mathrm{CFI}=0.963, \mathrm{RMSEA}=0.039)$. This questionnaire is a reliable and valid instrument and its use is suggested in future researches.
\end{abstract}

KEYWORDS: Physical Education; Infant Education; teachers; professional development; validation.

\title{
INTRODUCCIÓN
}

La motricidad, en su forma sistemática de la Educación Física (Gil- Madrona, Contreras-Jordán, Gómez-Víllora \& Gómez-Barreto, 2008) que presenta una gran importancia en el desarrollo global del infante en los primeros años de vida. Según Piaget y Buey (1969), a través de ella, se produce la formación de la personalidad y de los modos de conducta conformada por los dominios: afectivo, social, cognoscitivo y psicomotor (Gil-Madrona, Contreras, \& Gómez- Barreto, 2008). En relación con ello, diversos autores hacen referencia a los beneficios del desarrollo motriz en la primera infancia, tanto a largo como a corto plazo (Barnett, Van Beurden, Morgan, Brooks, \& Beard, 2008) sobre la personalidad y habilidades del niño en los citados dominios (Ward, 2010).

A través de la Educación Física Infantil se promueve el desarrollo motor, facilitando y afianzando la maduración referente al control del cuerpo (mantenimiento de postura, movimientos amplios y locomotrices, movimientos más precisos que permiten las modificaciones de acciones) y el proceso de representación del cuerpo y de las coordenadas espacio-temporales donde se desarrolla la acción (Delgado y Montes, 2017).

También el valor pedagógico de la Educación Física Infantil es de especial relevancia ya que promueve hábitos de vida saludable y activa mediante el desarrollo de movimientos motores básicos (Carson et al., 2015; Giles et al., 2017) y, además, debido a la conexión entre las capacidades cognitivas del infante y la actividad motora puesto que ambas capacidades progresan simultáneamente. En efecto, la Educación Física es el medio por el cual se pueden favorecer las funciones mentales (Davis et al., 2011) tales como la 
creatividad (Lupu, 2010), lo que la convierte en el medio más idóneo para propiciar y promover el desarrollo, evolución y adquisición de aprendizajes en Educación Infantil, mejorando el rendimiento académico (Shore et al., 2008). Otones y López-Pastor (2014), mediante un programa de motricidad aplicado al segundo ciclo de Educación Infantil, demostraron que la Educación Física en Educación Infantil fomenta la motivación, la atención del alumnado, la participación activa y el trabajo en valores. También las emociones son promovidas mediante la Educación Física debido al gran interés que sus actividades generan en Educación Infantil. Es por ello que, no solo propicia el desarrollo motor, sino que el niño se expresa y comunica lo que siente convirtiéndose socialmente más hábil (Gutiérrez, Fontenla, Cons, Rodríguez, \& Pazos, 2017; Pons \& Arufe, 2016).

Por tanto, la etapa de Educación Infantil es perfecta para la promoción de competencias específicas en el niño, que marcarán su personalidad y su posterior desarrollo tanto personal como académico (Silva, Neves, \& Moreira, 2016).

La promoción de estos beneficios depende de la labor de los maestros y educadores infantiles. A tal efecto, es preciso hablar sobre la relevancia del docente de Educación Infantil en relación con su desarrollo profesional y su vinculación con la Educación Física, ya que este actúa de manera muy significativa como eje en su desarrollo. Además, es el primer responsable de las prácticas que se llevan a cabo en Educación Física en esta etapa educativa (Sugrañes et al., 2007. Es por ello que requiere de unos conocimientos en relación con el contenido y metodología en el desarrollo de la Educación Física Infantil y a las características del alumnado de esta etapa (Moreno, López, Gutiérrez, Cascada, \& Fernández, 2004).

En este punto, debemos hablar de la necesidad de un cuestionario que evalúe el desarrollo profesional del docente de dicha etapa educativa en su vinculación con la Educación Física Si bien hemos encontrado distintos cuestionarios que evalúan esta temática, presentan algunas limitaciones.

Uno de ellos es el cuestionario elaborado por Sanz, Alonso, Valdemoros y Ponce de León (2013) cuya finalidad es evaluar el desarrollo profesional del profesorado de la etapa de infantil en relación a la educación para la salud. Dicho cuestionario se centra en el ámbito de la educación para la salud, por lo que no atiende al desarrollo de habilidades motoras, sociales, cognitivas y emocionales. El análisis factorial llevado a cabo pone de relieve que la fiabilidad interna es relativamente baja ya que algunos de los factores se encuentran en torno al ,0,6 y de acuerdo con González (2014), el valor de consistencia debería estar por encima del 0,7.

Otro instrumento es la Encuesta para profesorado de Infantil, elaborada por Latorre (2007). Se compone de 10 ítems, que hacen referencia a los aspectos de formación, perspectiva contextual y personal. No obstante, no se incluyen aquellos aspectos como son el reconocimiento social y la percepción del contexto acerca de la importancia de la Educación Física Infantil y su influencia en el desarrollo profesional del docente. Su fiabilidad es aceptable $(\alpha=0,706)$, 
aunque sería preferible que la consistencia interna se encuentre por encima del 0,8 (González, 2014).

En la misma línea, en el Cuestionario para el análisis de la Motricidad en Educación Infantil elaborado por Moreno et al. (2004) tampoco se hace referencia al reconocimiento social que percibe los maestros y educadores infantiles sobre su actividad docente en torno a la Educación Física infantil en la etapa de Educación Infantil, aunque su fiabilidad en este caso es buena ( $\alpha=$ 0,870) de acuerdo a la literatura (Huh, Delorme, \& Reid, 2006).

Ninguno de los instrumentos ha sido sometidos al Análisis Factorial Confirmatorio (AFC), sino que los análisis se han centrado en la búsqueda de relaciones subyacentes entre variables o constructos, sin partir de un modelo a priori entre dichas relaciones.

Así, el objetivo de esta investigación es construir y validar un instrumento de evaluación del desarrollo profesional de los docentes de Educación Infantil en su vinculación con la Educación Física, añadiendo el reconocimiento social y la percepción externa de la Educación Física ya que no se han teniendo en consideración en anteriores cuestionarios.

\section{MÉTODO}

\section{PARTICIPANTES}

En este estudio han participado 526 docentes en activo en la etapa 0-6 años, de la provincia de Albacete (España), de los cuales una muestra de 118 sujetos ha formado parte de la fase exploratoria del estudio y, posteriormente, se ha tomado una muestra de 408 docentes para participar en la fase final.

De los 118 participantes pertenecientes a la fase exploratoria, 14 son hombres $(11,9 \%)$ y 104 son mujeres $(88,1 \%)$, de edades comprendidas entre $25-65$ años $(M=43,75 ; D T=10,36)$. En la fase final, se contó con 408 participantes de edades comprendidas entre los 25-65 años $(M=43,37 ; D T=10,34)$, de los cuales $372(91,4 \%)$ son mujeres y $35(8,6 \%)$ son hombres. La distribución heterogénea de los participantes por sexo se debe a la elevada feminización existente en la profesión de docente en Educación Infantil (Junta de Comunidades de Castilla La Mancha, 2017). En su mayoría, los participantes desempeñan su labor en centros públicos $(N=308 ; 75,5 \%)$, siendo inferior la muestra que representa a los docentes de centros concertados-privados $(N=$ $100 ; 24,6 \%)$.

El criterio de selección fue el de acceso a la muestra mediante contacto con los centros educativos y la disposición de los profesionales a formar parte del estudio.

\section{INSTRUMENTO}

Se ha empleado el "Cuestionario para la evaluación del desarrollo profesional del maestro de Educación Infantil en relación a la Educación Física Infantil (CDP- 
EFI). Se trata de un cuestionario diseñado ad hoc, por expertos en Ciencias de la Educación (especialmente, de la Educación Física, en Educación Infantil y Primaria) y en Metodología de Investigación Educativa. En su fase inicial, el instrumento se compuso por 30 ítems, medidos en una escala tipo Likert de 5 puntos (de 1 = nada a 5 = mucho) y organizado en cinco bloques de contenido 0 dimensiones: desarrollo profesional, percepción laboral, perspectiva personal, percepción de la Educación Física infantil (externa e interna) y perspectiva contextual. Tras someterlo a un doble proceso de depuración (juicio de expertos y análisis factorial exploratorio, AFE), el instrumento quedó compuesto por 24 ítems (habiendo eliminado seis ítems del cuestionario inicial), distribuidos en 7 bloques de contenido o dimensiones:

1. Formación y desarrollo profesional: formación inicial y permanente del docente en torno a la Educación Física Infantil y dominio de dichos contenidos.

2. Valor formativo de la Educación Física Infantil: contribución de este ámbito a las distintas habilidades en la etapa 0-6 años.

3. Perspectiva personal: nivel de motivación de los maestros y educadores hacia la docencia de la Educación Física Infantil.

4. Percepción de la Educación Física Infantil (Externa): recursos materiales y elementos curriculares que condicionan a los docentes en la Educación Física Infantil.

5. Percepción de la Educación Física infantil (Interna): importancia concedida a la Educación Física en la etapa 0-6 años por parte del maestro.

6. Perspectiva contextual: valor concedido a la Educación Física Infantil por parte de las familias, compañeros maestros y educadores.

7. Reconocimiento social: consideración de los alumnos y familias sobre la actividad del docente de Educación Infantil en torno a la Educación Física Infantil.

\section{PROCEDIMIENTO}

El diseño y la validación del CDP-EFI se llevaron a cabo en varias fases:

Fase exploratoria. El punto de partida para la construcción del CDP-EFI fue un análisis de la literatura sobre desarrollo profesional del maestro de Educación Infantil, con especial hincapié en la Educación Física. EI CDP-EFI fue sometido a un doble proceso de depuración para asegurar su validez de contenido y su aplicabilidad:

- Juicio de expertos: se presentó a un grupo de cinco expertos en Educación Física Infantil de diferentes universidades españolas quienes 
señalaron algunas modificaciones en la redacción de los ítems que no afectaron a la estructura del instrumento.

- Estudio piloto. Se realizó un AFE y se comprobó la fiabilidad del instrumento para garantizar la validez de constructo. Para ello, se contó con una primera muestra de participantes $(N=118)$, que posteriormente fueron descartados para la aplicación del cuestionario modificado en la fase final del estudio.

Fase final. Tras eliminar algunos ítems que afectaban a la validez del instrumento, la versión definitiva quedó compuesta por 24 ítems. Esta versión fue aplicada a $\mathrm{N}=408$ participantes. Los datos obtenidos fueron sometidos a AFC y se estudió la fiabilidad compuesta y la varianza media extractada, para garantizar la validez de constructo del cuestionario final.

\section{ANÁLISIS DE DATOS}

Los datos fueron analizados con IBM SPSS 23.0 (Statistical Package for Social Sciences) y el programa AMOS 23. Antes de iniciar los análisis, se prepararon los datos para su tratamiento estadístico mediante la recodificación de ítems formulados en negativo (ítem 18) y la imputación de los valores perdidos. Dado que se trata de valores perdidos al azar, MAR (Missing at Random) predecibles a partir de los componentes observados de otras variables (Lang \& Little, 2016), se usó el método de imputación múltiple, mediante el cálculo de las estimaciones de regresión lineal múltiple. Además, se eliminaron casos de docentes cuyos cuestionarios presentaban más de $25 \%$ de respuestas sin contestar.

\section{RESULTADOS}

\section{ANÁLISIS FACTORIAL EXPLORATORIO DEL CDP_EFI INICIAL}

Se llevó a cabo un AFE por el método de factorización de ejes principales y rotación varimax sobre los resultados de la versión original del instrumento. Los resultados de la prueba de esfericidad de Bartlett $\left(X^{2} 435=1495,613 ; p>0,001\right)$ y la prueba de Kaiser-Meyer-Olkin $(K M O=0,718)$ permitieron descartar que las correlaciones entre ítems constituyesen una matriz de identidad. Asimismo, se tuvo en cuenta el valor del determinante de las correlaciones $(D=7,619)$, indicando bajas intercorrelaciones entre variables. Se analizó el grado de desviación de las puntuaciones de la distribución normal examinando la asimetría y la curtosis.

La estructura inicial extraída del AFE ofreció, inicialmente, una solución en 9 factores que explica el $57,34 \%$ de la varianza. Tras analizar el peso factorial de los ítems en cada factor, se observaron ítems con pesos inferiores a 0,4 que por tanto presentan un bajo poder explicativo de la dimensión que forman parte por lo que se eliminaron (ítems 4A, 4E, 9, 11, 18 y 22). Con ello, se obtuvo una solución en 7 factores que explicó un $59,12 \%$ de la varianza total. En la Tabla 1 se presenta los ítems del instrumento repartidos en las seis dimensiones 
extraídas del AFE, junto con su fiabilidad, siendo la fiabilidad total de la escala $\alpha$ $=0,836$. Esta estructura se tomó como base para el AFC.

Tabla 1. Resultado del AFE con rotación a siete factores: Dimensiones e ítems

\begin{tabular}{llc}
\hline Dimensión & Ítems & $A$ \\
\hline Valor formativo & I4B. Considero importantes las clases de EF en El porque & 0,783 \\
de la EFI & contribuye a: integración de niños de diversas culturas. & \\
& I4D. Considero importantes las clases de EF en El porque & \\
& contribuye a: adquisición de hábitos de salud. & \\
& I4G. Considero importantes las clases de EF en El porque & \\
& contribuye a: adquisición de hábitos y normas. & \\
& I4F. Considero importantes las clases de EF en El porque \\
& contribuye a: socialización. & \\
& I4C. Considero importantes las clases de EF en El porque \\
& contribuye a: expresión emocional y educación emocional.
\end{tabular}

Perspectiva 123. La EFI es considerada socialmente en esta etapa como una contextual actividad de segundo orden, "maría" o área débil por parte de los docentes.

124. La EFI es considerada socialmente en esta etapa como una actividad de segundo orden, María o área débil por parte de los padres.

112. La percepción que tengo de la docencia en EFI ha mejorado con respecto a cuando empecé a trabajar.

Formación y 17. Leo publicaciones de EF para niños de 0 a 6 años.

15. La formación inicial que recibí en la Formación Profesional o en la Universidad es suficiente y adecuada para trabajar la EF en mi escuela infantil o colegio.

18. Domino los contenidos de Educación Física para niños de 0 a 6 años.

Perspectiva personal

113. Me encuentro motivado e ilusionado cuando imparto clases de EFI.

115. Deseo impartir clase en otros ámbitos del currículum además de la EF.

114. Me gusta impartir docencia en EF a todos los grupos de alumnos de mi centro.

13. Me gusta impartir las clases de EFI a mis alumnos.

Percepción de I20. Elaboro una programación anual de EFI.

la EFI (Externa) 119. Cuento con los suficientes medios materiales, y éstos están en buen estado para impartir las clases de Educación Física Infantil.

121.Desarrollo y sigo la programación planificada.

Reconocimiento I16. Tengo el reconocimiento de mis actuaciones por parte de 0,769 los alumnos.

110. El nivel de motivación de los alumnos en las clases de EFI es alto. 
117. Tengo el reconocimiento de mis actuaciones por parte de los padres de mis alumnos.

Percepción de I1. Valor concedido a la EFI en el periodo de 0 a 3 años la EFI (Interna) 12. Valor concedido a la EFI en el periodo de 3 a 6 años. Nota: Educación Física (EF), Educación Infantil (EI), Educación Física Infantil (EFI)

\section{DESCRIPCIÓN DE LOS ÍTEMS DEL CDP-EMI FINAL}

En la Tabla 2 se presentan los índices de tendencia central y distribución que componen la evaluación del instrumento final, tras su aplicación a una muestra de 408 sujetos. De acuerdo con los valores obtenidos, la asimetría de los ítems fue negativa pero no excedió al valor -1 en la mayor parte de los casos. Para más del $70 \%$ de los ítems, la asimetría ocurrió en valores absolutos inferiores a 0,7 , lo que refierió ausencia de desviaciones significativas de la distribución normal. Con respecto a la curtosis, solo un elemento excedió los límites del intervalo $[-2,2]$. En lo que respecta a la curtosis multivariada, el coeficiente de Mardia obtuvo un valor de 125,735 , que resultó compatible con la multinormalidad del conjunto de variables observadas al situarse por debajo de $p^{*}(p+2)$, donde $p$ es el $n^{\circ}$ de variables.

Tabla 2. Estadísticos descriptivos para los ítems

\begin{tabular}{lrrrrr}
\hline Ítems & $N$ & $M$ & $D T$ & \multicolumn{1}{l}{ Asimetría } & \multicolumn{1}{c}{ Curtosis } \\
\hline I1 & 408 & 4,22 & 0,837 & $-0,714$ & 0,465 \\
I2 & 408 & 4,35 & 0,738 & $-1,018$ & 0,994 \\
I3 & 408 & 3,95 & 0,866 & $-0,896$ & 0,671 \\
I4B & 408 & 4,15 & 0,815 & $-0,688$ & 0,003 \\
I4C & 408 & 4,39 & 0,705 & $-0,799$ & 0,992 \\
I4D & 408 & 4,34 & 0,749 & $-0,655$ & 0,454 \\
I4F & 408 & 4,54 & 0,678 & $-1,004$ & 1,525 \\
I4G & 408 & 4,60 & 0,626 & $-0,734$ & 1,951 \\
I5 & 408 & 2,87 & 0,801 & 0,080 & $-0,703$ \\
I6 & 408 & 3,05 & 0,892 & $-0,199$ & $-0,868$ \\
I7 & 408 & 3,10 & 0,856 & $-0,225$ & $-0,809$ \\
I8 & 408 & 3,57 & 0,899 & $-0,447$ & 0,186 \\
I10 & 408 & 4,10 & 0,886 & $-0,524$ & 0,647 \\
I12 & 408 & 3,17 & 0,803 & -.315 & $-0,586$ \\
I13 & 408 & 3,85 & 0,632 & $-0,555$ & 0,535 \\
I14 & 408 & 3,38 & 0,971 & $-0,353$ & $-0,620$ \\
I15 & 408 & 3,87 & 0,983 & $-0,697$ & 0,180 \\
I16 & 408 & 4,05 & 0,928 & $-0,704$ & 0,576 \\
I17 & 408 & 3,57 & 0,809 & $-0,439$ & $-0,295$ \\
I19 & 408 & 3,24 & 0,927 & $-0,116$ & $-0,467$ \\
I20 & 4,46 & 0,858 & $-0,563$ & $-0,251$ \\
I21 & 408 & 3,46 & 0,064 \\
I23 & 408 & 3.76 & 0,927 & $-0,596$ & $-0,450$ \\
I24 & 408 & 3,01 & 1,041 & $-0,234$ & $-0,489$ \\
\hline & 408 & 3,10 & 1,059 & $0, .238$ &
\end{tabular}




\section{ANÁLISIS FACTORIAL CONFIRMATORIO}

Para confirmar la validez de constructo del instrumento, se realizó un AFC, empleando el método de máxima verosimilitud. No obstante, inicialmente se empleóa un modelo unifactorial, proponiendo la hipótesis nula $\left(\mathrm{H}_{0}\right)$ de que existe un solo factor en el que todos los ítems están saturados. Rechazar este modelo por falta de bondad de ajuste, supondría entender que resulta válido continuar explorando modelos con más factores. En el segundo modelo, se incluyeron los siete componentes. Cada elemento cargó solo en una variable latente, los factores covariados y los términos de error no correlacionaron.

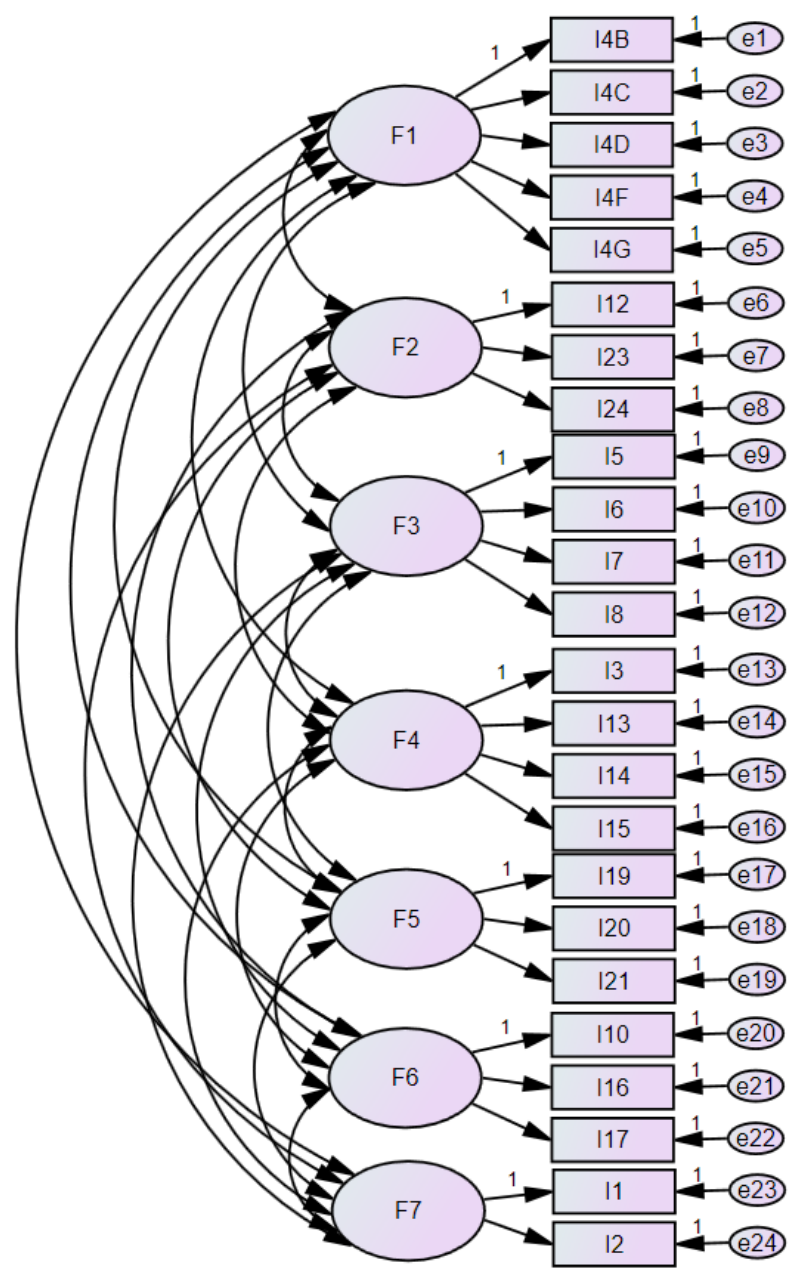

Figura 1. Estructura factorial del modelo en siete factores.

La Figura 1 muestra la estructura del modelo final (siete factores). Teniendo en cuenta los índices de modificación, se evaluó la importancia de los parámetros asociados con la covarianza entre los factores individuales, y se analizó la adecuación de los modelos en base a los índices de bondad de ajuste; concretamente, se tuvo en cuenta la relación entre $x 2$ y grados de libertad, el índice de ajuste comparativo (CFI), el índice de bondad de ajuste (GFI), el ajuste residual de raíz cuadrática media (RMR) y el error cuadrático medio de aproximación (RMSEA). También se estudiaron las correlaciones estandarizadas entre factores y entre variables y factores para confirmar, por un lado, la validez de constructo del instrumento $y$, por otro lado, la validez 
discriminante del instrumento, atendiendo a si la correlación entre las variables latentes, atenuadas por el error de medición (+/- 2 veces el error de medición), era menor que la unidad base.

\section{ESTRUCTURA FACTORIAL}

Los resultados presentados en la Tabla 3 indicaron la falta de ajuste del modelo unifactorial, permitiendo rechazar la unidimensionalidad de la escala y proponer la existencia de medidas diferenciadas para diferentes factores. Siguiendo los criterios propuestos por varios autores (Marsh, Hau, \& Wen, 2004; Ntoumanis, 2001), la bondad de ajuste fue mayor para el modelo final en siete factores. En dicho modelo, la relación entre $x 2$ y sus grados de libertad fue 1,635, un valor que no excedió el límite de 3, indicando un buen ajuste entre el modelo propuesto y los datos observados. El índice de ajuste comparativo (CFI) superó el nivel de 0,900, un valor considerado aceptable, al igual que el índice de bondad de ajuste (GFI), que excedió 0,900 en el modelo final (GFI =0,929), frente a un pobre ajuste observado en el modelo unifactorial ( $\mathrm{GFI}=0,621)$. El valor de RMR se mantuvo por debajo de 0,050 sólo en el caso del modelo final, lo que también indicó un mejor ajuste de éste. Finalmente, un error cuadrático medio de aproximación (RMSEA) por debajo de 0,080 se consideró aceptable, y los más cercanos a 0,050 se consideraron óptimos. De acuerdo con estos índices, el modelo de siete factores proporcionó el mejor ajuste.

Tabla 3. Índices de bondad de ajuste del modelo factoriales

\begin{tabular}{lccccc}
\hline Modelo & $\chi^{2} / \mathrm{gl}$. & CFI & GFI & RMR & RMSEA (CI 90\%) \\
\hline Unifactorial & 12,529 & 0,336 & 0,621 & 0,129 & $0,160(0,155-0,165)$ \\
Siete factores & 1,635 & 0,963 & 0,929 & 0,042 & $0,039(0,032-0,046)$ \\
\hline
\end{tabular}

Nota: $\mathrm{CFI}=$ Comparative Fit Index; GFI = Goodness of Fit Index; RMR = Root

Mean Square Residual; RMSEA = Root Mean Square Error of Approximation

La Tabla 4 presenta los coeficientes de correlación estandarizado entre factores y entre éstos y las variables y factores. Las cargas factoriales de cada variable obtuvieron valores adecuados, superiores a .70 en el $50 \%$ de los casos, y tan solo tres casos, por debajo de 0,60. Todos los ítems fueron estadísticamente significativos, con $p<0,01$. La carga más baja se situó en el ítem I17 (Tengo el reconocimiento de mis actuaciones por parte de los padres de mis alumnos) con un valor de 0,528 en el Factor 6 "Reconocimiento social". El más alto fue 0,966 en el ítem 2 (Valor concedido a la Educación Física en la Educación Infantil en el periodo de 3 a 6 años) en el Factor 7 "Percepción de la EFI (Interna)" es adecuado. Los resultados obtenidos apoyaron la estructura de siete factores propuesta para el instrumento y, por lo tanto, implicaron validez de constructo. 
Tabla 4. Cargas factoriales para el modelo en siete factores, incluyendo las correlaciones entre términos de error

\begin{tabular}{|c|c|c|}
\hline Items & Load & $\mathrm{R}^{2}$ \\
\hline \multicolumn{3}{|l|}{ Valor formativo de la EFI } \\
\hline $\begin{array}{l}\text { 14B. Considero importantes las clases de EF en El porque contribuye a: } \\
\text { integración de niños de diversas culturas }\end{array}$ & 0,611 & 0,416 \\
\hline $\begin{array}{l}\text { 14D. Considero importantes las clases de EF en El porque contribuye a: } \\
\text { adquisición de hábitos de salud }\end{array}$ & 0,633 & 0,336 \\
\hline $\begin{array}{l}\text { I4G. Considero importantes las clases de EF en El porque contribuye a: } \\
\text { adquisición de hábitos y normas. }\end{array}$ & 0,590 & 0,228 \\
\hline $\begin{array}{l}\text { I4F. Considero importantes las clases de EF en El porque contribuye a: } \\
\text { socialización }\end{array}$ & 0,709 & 0,255 \\
\hline $\begin{array}{l}\text { 14C. Considero importantes las clases de EF en El porque contribuye a: } \\
\text { expresión emocional y educación emocional }\end{array}$ & 0,720 & 0,239 \\
\hline \multicolumn{3}{|l|}{ Perspectiva contextual } \\
\hline $\begin{array}{l}\text { 123. La EFI es considerada socialmente en esta etapa como una actividad de } \\
\text { segundo orden, "maría" o área débil por parte de los docentes }\end{array}$ & 0,939 & 0,128 \\
\hline $\begin{array}{l}\text { 124. La EFI es considerada socialmente en esta etapa como una actividad de } \\
\text { segundo orden, María o área débil por parte de los padres }\end{array}$ & 0,943 & 0,124 \\
\hline $\begin{array}{l}\text { 112. La percepción que tengo de la docencia en EFI ha mejorado con respecto } \\
\text { a cuando empecé a trabajar }\end{array}$ & 0,906 & 0,216 \\
\hline \multicolumn{3}{|l|}{ Formación y desarrollo profesional } \\
\hline 17. Leo publicaciones de EF para niños de 0 a 6 años. & 0,624 & 0,515 \\
\hline $\begin{array}{l}\text { 16. Recibo formación (cursos, congresos, jornadas...) específica de EF para } \\
\text { niños de } 0 \text { a } 6 \text { años. }\end{array}$ & 0,627 & 0,559 \\
\hline $\begin{array}{l}\text { 15. La formación inicial que recibí en la Formación Profesional o en la } \\
\text { Universidad es suficiente y adecuada para impartir en el ámbito de EF en mi } \\
\text { escuela infantil o colegio. }\end{array}$ & 0,588 & 0,491 \\
\hline $\begin{array}{l}\text { 18. Domino los contenidos de la EF para niños de } 0 \text { a } 6 \text { años. } \\
\text { Perspectiva personal }\end{array}$ & 0,678 & 0,435 \\
\hline 113. Me encuentro motivado e ilusionado cuando imparto clases de EFI & 0,840 & 0,254 \\
\hline $\begin{array}{l}\text { l15. Deseo impartir clase en otros ámbitos del currículum además de trabajar } \\
\text { la EFI }\end{array}$ & 0,671 & 0,529 \\
\hline $\begin{array}{l}\text { I14. Me gusta impartir docencia en EFI a todos los grupos de alumnos de mi } \\
\text { centro }\end{array}$ & 0,620 & 0,542 \\
\hline $\begin{array}{l}\text { I3. Me gusta impartir las clases de EFI a mis alumnos } \\
\text { Percepción de la EFI (Externa) }\end{array}$ & 0,804 & 0,265 \\
\hline 120. Elaboro una programación anual de EFI & 0,705 & 0,561 \\
\hline $\begin{array}{l}\text { 119. Cuento con los suficientes medios materiales, y éstos están en buen } \\
\text { estado para impartir las clases de EFI }\end{array}$ & 0,666 & 0,585 \\
\hline $\begin{array}{l}\text { I21.Desarrollo y sigo la programación planificada. } \\
\text { Reconocimiento social }\end{array}$ & 0,752 & 0,372 \\
\hline 116. Tengo el reconocimiento de mis actuaciones por parte de los alumnos & 0,859 & 0,225 \\
\hline 110. El nivel de motivación de los alumnos en las clases de EFI es alto. & 0,847 & 0,221 \\
\hline $\begin{array}{l}\text { 117. Tengo el reconocimiento de mis actuaciones por parte de los padres de } \\
\text { mis alumnos. }\end{array}$ & 0,528 & 0,433 \\
\hline \multicolumn{3}{|l|}{ Percepción de la EFI (Interna) } \\
\hline 11. Valor concedido a la EFI en el periodo de 0 a 3 años & 0,604 & 0,444 \\
\hline 12. Valor concedido a la EFI en el periodo de 3 a 6 años & 0,966 & 0,037 \\
\hline
\end{tabular}

Nota: $R 2$ = Porcentaje de varianza explicada.

Nota: Educación Física (EF), Educación Infantil (EI), Educación Física Infantil (EFI)

La matriz de correlaciones entre factores se presenta en la Tabla 5. Los valores alcanzaron valores promedio, entre 0,007 (correlación negativa entre perspectiva 
contextual y perspectiva personal) y 0,733 (correlación entre perspectiva contextual y formación y desarrollo profesional). Las bajas correlaciones entre factores descartaron la posibilidad de que dos factores representasen la misma dimensión. Esto respaldó la validez discriminante del instrumento, mostrando dimensiones suficientemente diferentes. También cabe destacar la existencia de dimensiones que correlacionaron en negativo, como por ejemplo perspectiva contextual y percepción personal $(r=-0,007)$ y percepción interna de la Educación Física infantil y perspectiva contextual $(r=-0,024)$.

Tabla 5. Matriz de correlaciones entre factores

\begin{tabular}{lccccccc}
\hline & VFMI & PC & FDP & PP & RS & PMIE & PMII \\
\hline VFMI & & & & & & & \\
PC & $-0,015$ & & & & & & \\
FDP & 0.162 & 0,733 & & & & & \\
PP & 0,226 & $-0,007$ & 0,530 & & & & \\
RS & 0,088 & 0,058 & 0,524 & 0,412 & & & \\
PMIE & 0,281 & -0.022 & 0,332 & 0,529 & 0,460 & & \\
PMII & 0,328 & $-0,108$ & 0,148 & 0,222 & 0,130 & 0,129 & \\
\hline
\end{tabular}

Nota: Valor Formativo EFI (VFEFI), Perspectiva Contextual (PC) Formación y Desarrollo Profesional (FDP), Perspectiva Personal (PP), Reconocimiento Social (RS), Percepción Educación Física Infantil Externa (PEFI), Percepción Educación Física Infantil Interna (PEFI)

\section{FIABILIDAD}

La fiabilidad del instrumento, presentada en la Tabla 6, se estimó a partir del Índice de Fiabilidad Compuesta (IFC) que permitió analizar todos los constructos implicados en la escala, y varianza media extractada (en inglés, Average Variance Extracted, AVE), como medida de validez convergente, en referencia al grado en que los indicadores miden de un modo preciso el constructo. Los valores obtenidos fueron, a nivel general, aceptables, siendo excelente en el caso del factor "Perspectiva contextual" e insuficiente para el caso de "reconocimiento social".

Tabla 6. Subescalas del alfa de Cronbach

\begin{tabular}{lccc}
\hline Factores & $\begin{array}{c}\text { Número } \\
\text { ítems }\end{array}$ & IFC & AVE \\
\hline Valor Formativo EFI & 5 & 0,593 & 0,429 \\
Perspectiva Contextual & 3 & 0,847 & 0,864 \\
Formación y desarrollo & 4 & 0,452 & 0,397 \\
profesional & & & \\
Perspectiva Personal & 4 & 0,579 & 0,547 \\
Reconocimiento social & 3 & 0,498 & 0,502 \\
Percepción EFI (Externa) & 3 & 0,664 & 0,578 \\
Percepción EFI (Interna) & 2 & 0,732 & 0,649 \\
\hline
\end{tabular}

\section{DISCUSIÓN}

El objetivo de esta investigación fue el diseño y validación del CDP-EMI mediante un AFE inicial con una muestra de 118 participantes en el estudio piloto, y posteriormente, a través de un AFC aplicado a una muestra final, de 408 participantes. En términos generales, los resultados evidencian que es un 
instrumento adecuado para evaluar el desarrollo profesional de los docentes de Educación Infantil en su vinculación con la Educación Física.

La estructura factorial exploratoria extrajo siete dimensiones, que explicaron el $59,12 \%$ de la varianza total: valor formativo de la EFI, perspectiva contextual, formación y desarrollo profesional, perspectiva personal, percepción interna de la EFI, perspectiva externa de la EFI y reconocimiento social. Dicha estructura factorial fue posteriormente confirmada, garantizándose una validez y fiabilidad adecuada en todas las dimensiones, con especial relevancia en la dimensión perspectiva contextual.

Respecto a las dimensiones de valor formativo de la EFI y percepción interna cabe señalar que, su importancia para evaluar la contribución de la EFI al desarrollo global del niño y el valor otorgado a la EFI por parte del docente, respectivamente, se ha hecho evidente en investigaciones precedentes como las de Latorre (2007), Moreno et al. (2004) y Sanz-Arazuri et al. (2013).

Lo mismo ocurre con las dimensiones formación y desarrollo profesional, con la que evaluamos la formación inicial y continua, la perspectiva personal, que mide la motivación docente, y la perspectiva externa de la EFI, con la que se estudian los recursos materiales, elaboración y seguimiento de la programación, todas ellas estudiadas previamente en Latorre (2007) o Valdemoros, Sanz, Ponce de León y Alonso (2018).

Sin embargo, las dimensiones más vinculadas a una perspectiva social de la EFI, tales como la perspectiva contextual, referida al valor que otorgan las familias y compañeros docentes, y reconocimiento social de las familias y alumnos sobre la actividad que desempeña estos maestros y educadores infantiles, suponen una novedad respecto a otros estudios sobre desarrollo profesional de los docentes (Latorre, 2007; Moreno et al., 2004; Sanz-Arazuri et al., 2013). Es, precisamente, la introducción de estas dimensiones, junto con la exploración de la relación entre las variables, su contraste y la evaluación del ajuste estadístico del modelo teórico propuesto, lo que otorga relevancia a instrumento.

\section{CONCLUSIÓN}

El CDP-EMI ofrece una medida global, fiable y válida, del desarrollo profesional de los docentes en su vinculación con la Educación Física Infantil. Dicho instrumento aportará información relevante para poder tomar decisiones acerca de la actividad docente en torno a la Educación Física Infantil y cuyos resultados se plasmarán en el proceso de enseñanza- aprendizaje en la etapa 0-6 años.

No obstante, este estudio no está exento de limitaciones. Cabe destacar las limitaciones a nivel muestral, teniendo en cuenta que la localización geográfica de la muestra se restringe a la Comunidad Autónoma de Castilla La Mancha. De ahí que, como perspectivas de futuro, se pretende replicar el estudio con una muestra representativa en el ámbito español y en el ámbito internacional. 


\section{REFERENCIAS BIBLIOGRÁFICAS}

Barnett, L. M., Van Beurden, E., Morgan, P. J., Brooks, L .O., \& Beard, J. R. (2008). Does childhood motor skill proficiency predict adolescent fitness?. Medicine \& Science in Sports \& Exercise, 40(12), 2137-2144. doi: https://doi.org/10.1249/MSS.0b013e31818160d3

Carson, V., Kuzik, N., Hunter, S., Wiebe, S.A., Spence, J. C., Friedman, A., ... \& Hinkley, T. (2015). Systematic review of sedentary behavior and cognitive development in early childhood. Preventive Medicine, 78, 115-122. doi: https://doi.org/10.1016/j.ypmed.2015.07.016

Junta de Comunidades de Castilla La Mancha (2017). Estadística, 2016-2017. Avance de Datos. Castilla La Mancha: España. Portal de Educación de Castilla La Mancha. Recuperado de http://www.educa.jccm.es/es/consejeria-educacion-culturadeportes/estadistica-educativa/estadistica-2016-2017-avance-datos

Davis, C. L., Tomporowski, P. D., McDowell, J. E., Austin, B. P., Miller, P. H., Yanasak, N. E.,... \& Naglieri, J. A. (2011). Exercise improves executive function and achievement and alters brain activation in overweight children: a randomized, controlled trial. Health Psychology, 30(1), 91-98. doi: https://doi.org/10.1037/a0021766

Delgado, L., \& y Montes, R. (2017). Perfil y desarrollo psicomotor de los niños españoles entre 3 y 6 años. Sportis. Revista Técnico-Científica del Deporte Escolar, Educación Física y Psicomotricidad, 3(3), 454-470. doi: https://doi.org/10.17979/sportis.2017.3.3.2002

Gil-Madrona, P., Contreras, O.R., \& Gómez-Barreto, I. (2008). Habilidades motrices en la infancia desde una educación física animada. Revista Iberoamericana de Educación, 47, 71-96. Recuperado de https://dialnet.unirioja.es/servlet/articulo?codigo $=2736759$

Gil-Madrona, P., Gómez-Villora, S., Contreras-Jordán, O. R., \& Gómez-Barreto, I. (2008). Justificación de la educación física en la educación infantil. Educación y educadores, 11(2), 159-177. Recuperado de http://www.scielo.org.co/scielo.php?script=sci_abstract\&pid=S012312942008000200010

Giles, G. E., Cantelon, J. A., Eddy, M.D., Brunye, T.T., Urry, H.L., Mahoney, C.R., \& Kanarek, R.B. (2017). Habitual exercise is associated with cognitive control and cognitive reappraisal success. Experimental Brain Research, 235(12), 3785-3797. doi: https://doi.org/10.1007/s00221-017-5098-x

González, A. (2014). Características técnicas de los instrumentos de medida desde la teoría clásica de los tests: fiabilidad, validez. En C. Martínez (Ed.), Técnicas e instrumentos de recogida y análisis de datos (pp. 214240). Madrid: UNED.

Gutiérrez, L., Fontenla, E., Cons, M., Rodríguez, J.E., \& Pazos, J.M. (2017). Mejora de la autoestima y de la inteligencia emocional a través de la psicomotricidad y de talleres de habilidades sociales. Sportis. Revista Técnico-Científica del Deporte Escolar, Educación Física y Psicomotricidad, 3(1), 187-205. doi: https://doi.org/10.17979/sportis.2017.3.1.1813

Huh, J., DeLorme, D. E., \& Reid, L.N. (2005). Factors affecting trust in on-line prescription drug information and impact of trust on behavior following exposure to DTC advertising. Journal of Health Communication, 10(8), 711-731. doi: https://doi.org/10.1080/10810730500326716 
Lang, K. M., \& Little, T. D. (2016). Principled missing data treatments. Prevention Science, 1-11. doi: https://doi.org/10.1007/s11121-016-0644-5

Latorre, P.A. (2007). La motricidad en Educación Infantil, grado de desarrollo y compromiso docente. Revista Iberoamericana de Educación, 7(43), 1-7. Recuperado de https://dialnet.unirioja.es/servlet/articulo?codigo=2358750

Lupu, E. (2010). Cognition as an efficient way of training in physical education activities. Procedia-Social and Behavioral Sciences, 5, 2133-2139. doi: https://doi.org/10.1016/j.sbspro.2010.07.426

Marsh, H. W., Hau, K. T., \& Wen, Z. (2004). In search of golden rules: Comment on hypothesis-testing approaches to setting cutoff values for fit indexes and dangers in overgeneralizing. Structural Equation Modeling: $A$ Multidisciplinar Journal, 11(3), 320-341. doi: https://doi.org/10.1207/s15328007sem1103 2

Moreno, J. A., López, B., Gutiérrez, E.M., Cascada, M., \& Fernández, M.R. (2004). Situación actual de la motricidad en la etapa de 0 a 6 años según el profesorado de Educación Infantil. Revista Iberoamericana de Psicomotricidad y Técnicas Corporales, 16, 17-30. Recuperado de http://www.um.es/univefd/motinfantil.pdf

Ntoumanis, N. (2001). A self-determination approach to the understanding of motivation in physical education. British Journal of Educational Psychology, 71(2), 225-242. doi: https://doi.org/10.1348/000709901158497

Otones, R., \& López-Pastor, V. (2014). Un programa de cuentos motores para trabajar la motricidad en educación infantil. Resultados encontrados. Revista de Educación Física para la paz, 9, 27-44. Recuperado de https://dialnet.unirioja.es/servlet/articulo?codigo $=4746783$

Piaget, J., \& Buey, F. F. (1969). Psicología y pedagogía. Barcelona: Ariel.

Pons, R., \& Arufe, V. (2016). Análisis descriptivo de las sesiones e instalaciones de psicomotricidad en el aula de educación infantil. Sportis. Revista Técnico-Científica del Deporte Escolar, Educación Física y Psicomotricidad,2(1),125-146. https://doi.org/10.17979/sportis.2016.2.1.1445

Sanz, E., Alonso, R. A., Valdemoros, M., \& Ponce de León, A. (2013). Validación de un cuestionario que analiza cómo trabaja el profesorado de la etapa infantil la educación para la salud desde el ámbito motor. Revista Iberoamericana de Diagnóstico y Evaluación-e Avaliação Psicológica, 1(35), 9-34. Recuperado de http://www.redalyc.org/pdf/4596/459645435002.pdf

Shore, S.M., Sachs, M.L., Lidicker, J.R., Brett, S. N., Wright, A..R., \& Libonati, J.R. (2008). Decreased scholastic achievement in overweight middle school students. Obesity 16(7), 1535-1538. doi: https://doi.org/10.1038/oby.2008.254

Silva, M., Neves, G., \& Moreira, S. (2016). Efectos de un programa de psicomotricidad educativa en niños en edad preescolar. Sportis. Revista Técnico-Científica del Deporte Escolar, Educación Física y Psicomotricidad, 2(3), 326-342. doi: https://doi.org/10.17979/sportis.2016.2.3.1563

Sugrañes, E., Ángel, M. A. (Coords.), Andrés, M. N., Colomé, J., Martí, M. T., Martín, R. M.,... Yuste, R. (2007). La educación psicomotriz (3-8 años). Cuerpo, movimiento, percepción, afectividad: una propuesta teóricopráctica. Barcelona: Graó. 
Valdemoros, M. Á., Sanz, E., Ponce de León, A., \& Alonso, R. A. (2018). Cualificación e implicación del profesorado de infantil frente a la educación motriz. Sportis. Scientific Journal of School Sport, Physical Education and Psychomotricity, 4(1), 126-143. doi: https://doi.org/10.17979/sportis.2018.4.1.3165

Ward, D. S. (2010). Physical activity in young children: The role of child care. Medicine and Science in Sports y Exercise, 42(3), 499-501. doi: https://doi.org/10.1249/MSS.0b013e3181ce9f85

Número de citas totales/ Total references: 28 (100\%)

Número citas propias de la revista/ Journal's own references: $0(0 \%)$

Rev.int.med.cienc.act.fís.deporte - vol. 20 - número 77 - ISSN: 1577-0354 